\title{
Decoration of Carbon Nanotubes with CoO and Co Nanoparticles
}

\author{
M. Belesi, ${ }^{1,2}$ I. Panagiotopoulos, ${ }^{3}$ S. Pal, ${ }^{4}$ S. Hariharan, ${ }^{4}$ D. Tsitrouli, ${ }^{1}$ G. Papavassiliou, ${ }^{1}$ \\ D. Niarchos, ${ }^{1}$ N. Boukos, ${ }^{1}$ M. Fardis, ${ }^{1}$ and V. Tzitzios ${ }^{1}$ \\ ${ }^{1}$ Institute of Materials Science, N.C.S.R. "Demokritos" Agia Paraskevi, 15310 Athens, Greece \\ ${ }^{2}$ ICMP, Ecole Polytechnique Fédérale de Lausanne (EPFL), Station 3, 1015 Lausanne, Switzerland \\ ${ }^{3}$ Department of Materials Science and Engineering, University of Ioannina, 45110 Ioannina, Greece \\ ${ }^{4}$ Department of Physics, University of South Florida, Tampa, FL 33620, USA \\ Correspondence should be addressed to M. Belesi, mariaeleni.belesi@epfl.ch and V. Tzitzios, tzitzios@ims.demokritos.gr
}

Received 1 September 2010; Revised 15 December 2010; Accepted 27 January 2011

Academic Editor: Gaurav Mago

Copyright ( $) 2011 \mathrm{M}$. Belesi et al. This is an open access article distributed under the Creative Commons Attribution License, which permits unrestricted use, distribution, and reproduction in any medium, provided the original work is properly cited.

\begin{abstract}
Multiwall carbon nanotubes (MWNTs) decorated with $\mathrm{CoO}$ nanocrystals were synthesized by in-situ thermal decomposition of $\mathrm{Co}(\mathrm{acac})_{2}$ in oleyl amine under reflux conditions open in the air. The CoO/MWNTs composite material can be easily converted to metallic $\mathrm{Co} / \mathrm{MWNTs}$ through annealing under reducing atmosphere $\left(4 \% \mathrm{H}_{2}\right)$ at $500^{\circ} \mathrm{C}$ without any significant sintering effect. The composite materials characterized by X-ray diffraction, transmission electron microscopy, and Nuclear Magnetic Resonance (NMR) spectroscopy. The structural and morphological characterization shows that the CoO has cubic face $(f c c)$ and the particles deposited uniformly on the external surface of the carbon nanotubes. In the annealed materials, the NMR shows that the $f c c$ and hcp metallic Co phases coexist with a significant percentage of stacking faults. The magnetic measurements indicated that the $\mathrm{CoO} / \mathrm{MWNTs}$ composite is largely composed of $\mathrm{CoO}$ nanoparticles with uncompensated surface spins. The fluctuations of spins persist in partially reduced $\mathrm{CoO}$ grains as shown by nuclear spin-lattice relaxation measurements.
\end{abstract}

\section{Introduction}

In the past several years, there has been a growing interest in synthesizing carbon nanotube- (CNT-) inorganic nanocrystals composites including magnetic, semiconducting, and optic materials [1]. Due to their high specific surface area, electrical conductivity, and chemical stability, carbon nanotube nanohybrids are assumed ideal materials for catalytic applications, from fuel cells to heterogeneous catalysis, for hydrogen storage and finally for chemical and biochemical sensing applications. Among inorganic materials, magnetic particles have been studied more extensively for the functionalization of carbon nanotubes. This is related to their promising applications in the fields of nanoprobes for magnetic force microscopy, wastewater treatment, biosensors, and drug delivery [2-5]. Several approaches, including in situ decoration in liquid phase $[6,7]$, electrochemical reduction [8], electrophoretic deposition [9, 10], vapourliquid-solid (VLS) deposition [11], substrate-enhanced electrochemical reduction, nanoparticles decoration on chemically oxidized CNT, or chemically functionalized side walls
[12-17] have been attempted to prepare CNT-inorganic nanocrystals composites. In recent years cobalt and its oxide nanostructures have attracted much attention because they show interesting magnetic properties which may find potential applications in magnetic sensors and data storage devices.

In this work, we describe the decoration of carbon nanotubes with $\mathrm{CoO}$ nanoparticles and the transformation of $\mathrm{CoO}$ in metallic cobalt through thermal treatment under reducing atmosphere. The method is facile, based on environmental friendly raw materials, inexpensive, and reproducible for the large-scale synthesis of analogous composite nanomaterials. These hybrid structures may combine the applications of the bare nanotubes structures and potentially enhance their properties giving considerable impact on the potential applications of nanotubes. For example, the decoration of carbon nanotubes with Co-based oxides [18] has been shown to enhance the electrochemical capacitance when the composite is used as an electrode material. These composite materials may also show enhanced catalytic properties for industrial applications, or they can be used 


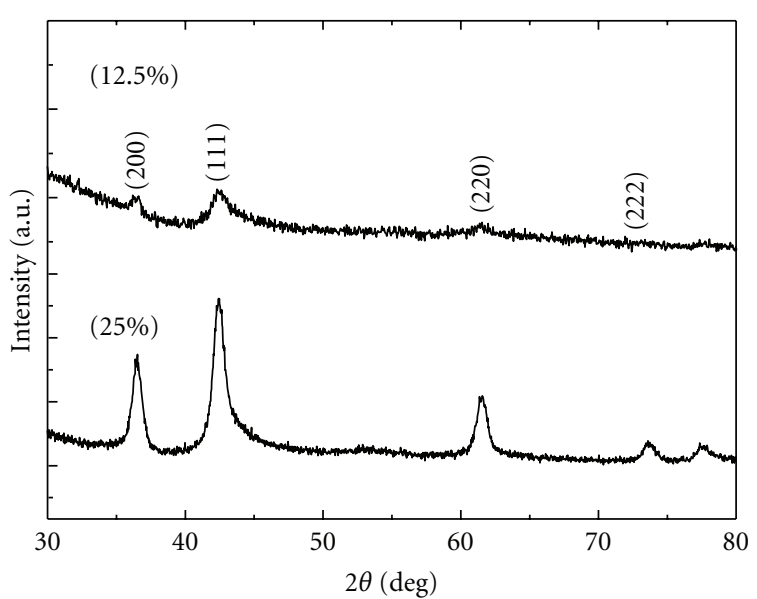

(a)

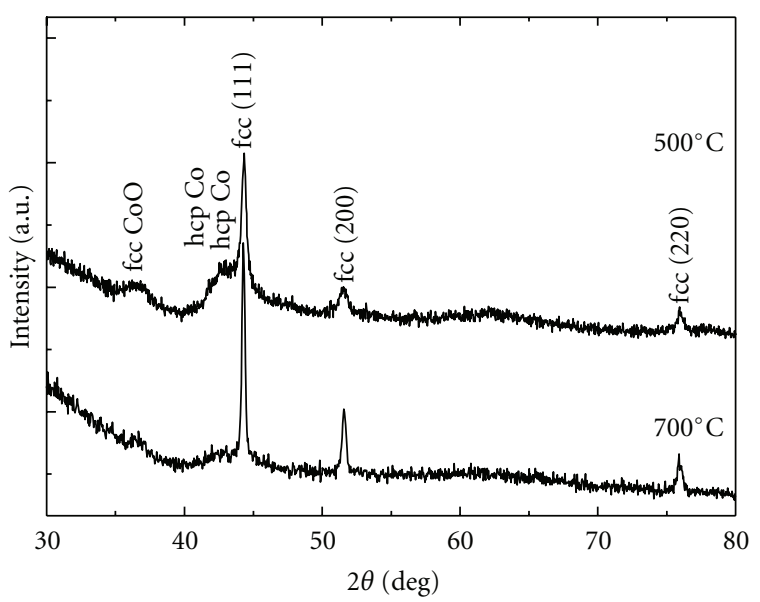

(b)

FIGURE 1: XRD patterns from as-made CoO/MWNTs composites with different nanoparticles loading (a) and after annealing under reducing $\mathrm{H}_{2}$-Ar atmosphere at $500^{\circ} \mathrm{C}$ and $700^{\circ} \mathrm{C}(\mathrm{b})$.

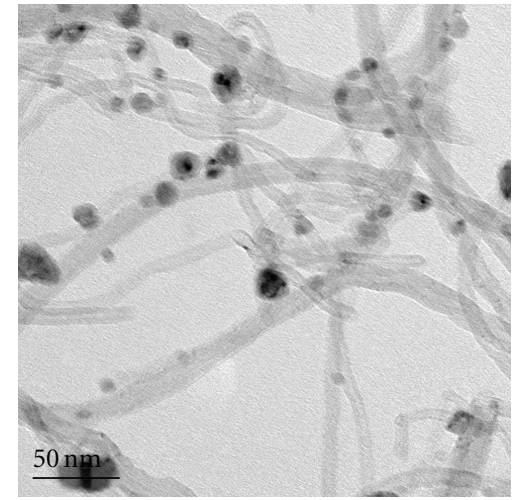

(a)

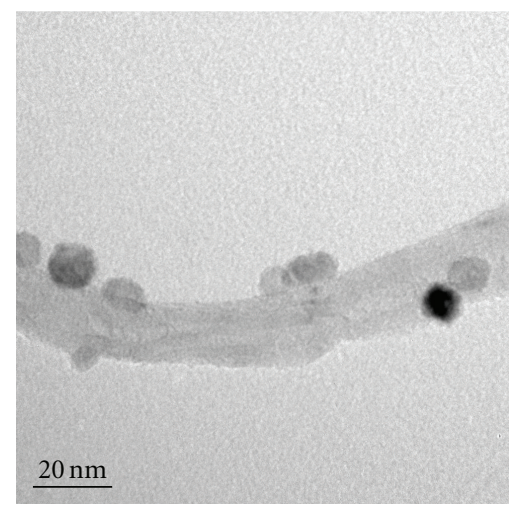

(c)

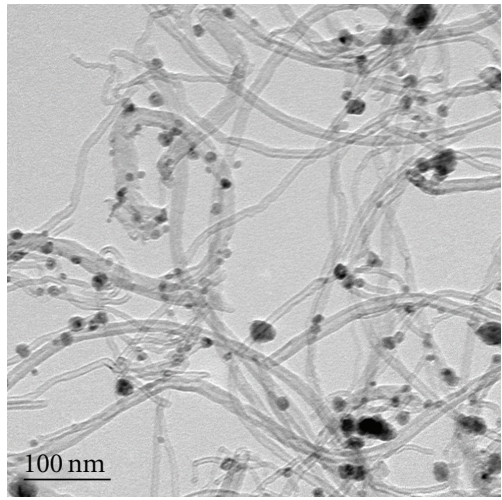

(b)

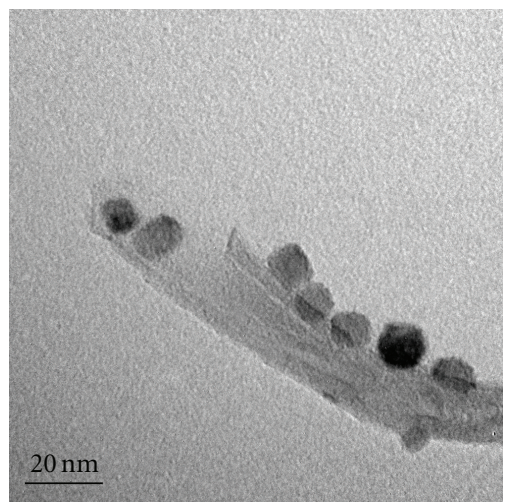

(d)

Figure 2: TEM images of $25 \mathrm{wt} \% \mathrm{CoO} / \mathrm{MWNTs}$ nanocomposites.

for electronic devices, biosensors, magnetic data storage devices, toners and inks for xerography, and also in magnetic resonance imaging.

We show here through magnetic characterization that the as-synthesized sample is composed of $\mathrm{CoO}$ nanoparticles. In the annealed samples, the presence of ferromagnetic Co is detected by magnetic and NMR measurements. Furthermore, the NMR measurements demonstrate the allotropic phase transformation of nanometric Co particles in the annealed samples and provide evidence for slow fluctuations of uncompensated spins in $\mathrm{CoO}$ nanoparticles which are still present in the sample annealed at $500^{\circ} \mathrm{C}$. 


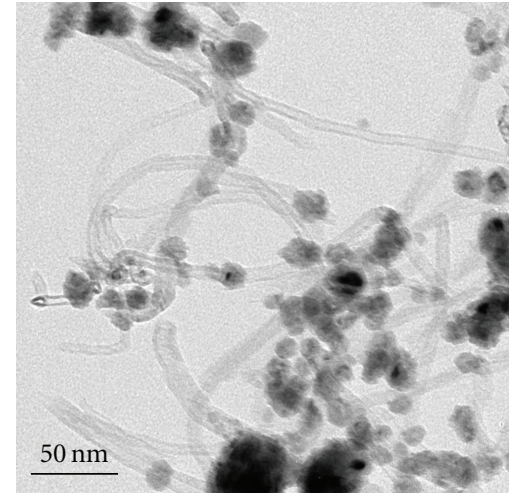

(a)

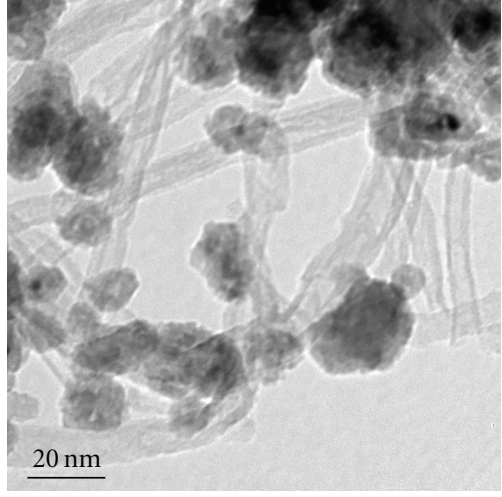

(b)

Figure 3: TEM images of $25 \mathrm{wt} \% \mathrm{CoO} / \mathrm{MWNTs}$ composite material after thermal treatment at $500^{\circ} \mathrm{C}$ under $5 \% \mathrm{H}_{2} 95 \%$ Ar atmosphere. Average size $20 \mathrm{~nm}$ with the small 5-6 nm.

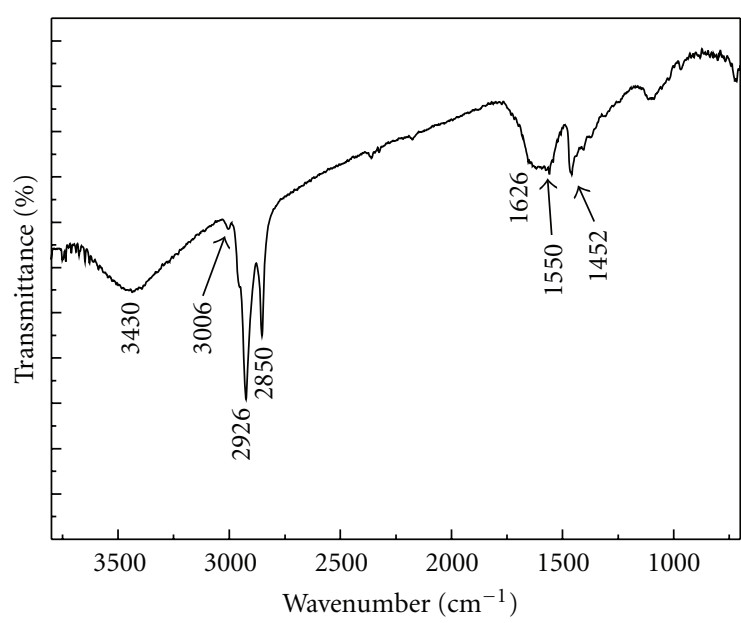

(a)

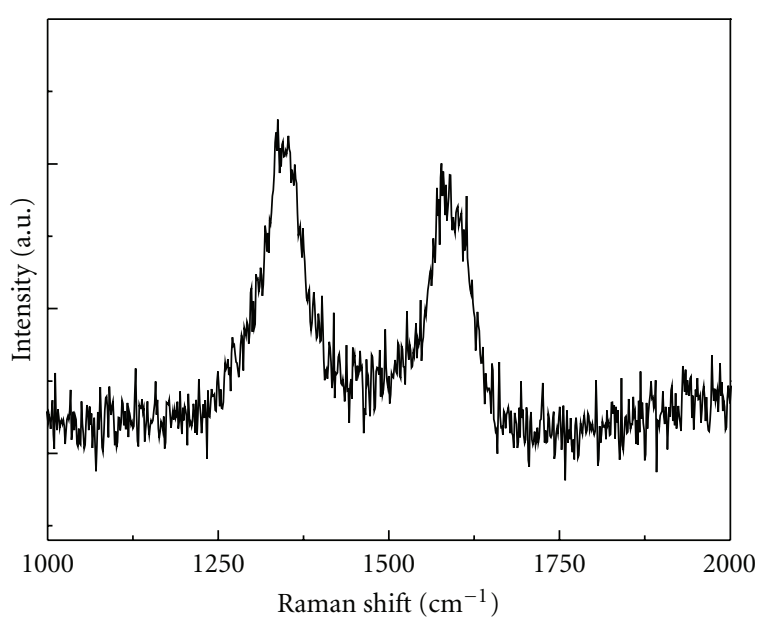

(b)

FIgURE 4: FT-IR spectrum from the as-made CoO/MWNTs composite material (a) and Raman spectrum from annealed at $500^{\circ} \mathrm{C}$ CoO/MWNTs composite material (b).

\section{Experimental Section}

It is well known that the thermolytic decomposition of various inorganic salts in oleyl amine leads to the formation of the corresponding oxide and in some cases in metallic nanoparticles due to the mild reduction properties of aliphatic amines [19]. The decoration of MWNT with CoO nanocrystals was carried out in a spherical flask under reflux conditions. The flask is charged with a mixture of $20 \mathrm{~mL}$ of oleyl amine, $200 \mathrm{mg}$ MWNT, and the mixture was sonicated for $5 \mathrm{~min}$ in order to achieve a good dispersion. Following, the mixture was heated at $100^{\circ} \mathrm{C}$ and $0.25-$ $0.75 \mathrm{mmol}$ of $\mathrm{Co}(\mathrm{acac})_{2}$ in $5 \mathrm{~mL}$ oleyl amine were added dropwise under vigorous magnetic stirring. The reaction completed at the reflux point for $1 \mathrm{~h}$ opened to air. After cooling the reaction mixture at room temperature, the crude solution was separated by centrifugation and the composite material was washed several times with ethanol in order to remove the oleyl amine excess and the byproducts. Finally, the washed $\mathrm{CoO} / \mathrm{MWNT}$ materials were dispersed in few $5 \mathrm{~mL}$ ethanol and dried at room temperature by spreading on a glass plate. The annealing of the as-made materials carried out at under continuous flow of $4 \% \mathrm{H}_{2}-96 \%$ Ar gas mixture.

The crystal structure of the composite materials was characterized by X-ray powder diffraction (XRD) (Siemens D $500 \mathrm{Cu} \mathrm{Ka}$ radiation). The morphology was investigated by using transmission electron microscopy (TEM). The annealed materials were additionally characterized by NMR spectroscopy. Magnetic properties were measured using a commercial Physical Properties Measurement System (PPMS) from Quantum Design.

\section{Results and Discussion}

3.1. Structural and Morphological Characterization. The XRD patterns from the as-made materials with different CoO loading provided in Figure 1(a) clearly show pure nanocrystalline cubic phase of the CoO. The diffraction 
peaks of the cubic phase are well matched with those of the corresponding bulk $\mathrm{CoO}(F m 3 m, \alpha=4.261 \AA)$ while the peaks are slightly broadened due to the nanocrystalline size. The calculated lattice parameter based on (200) peak is $4.265 \AA$, which is in very good agreement with the standard value of bulk $f c c \mathrm{CoO}$. From the XRD patterns, there is no evidence for the presence of other oxide phases such as $h c p$ $\mathrm{CoO}$ or $\mathrm{Co}_{3} \mathrm{O}_{4}$. Also although the oleyl amine is well-known mild reducing agent, there are no metallic Co traces because the synthetic reaction takes place under air conditions. Figure 1(b) shows the XRD patterns from the 25\% $\mathrm{CoO}$ loading at 500 and $700^{\circ} \mathrm{C}$. The sample that annealed at $500^{\circ} \mathrm{C}$ shows the presence of $f c c$ metallic Co which is the main phase but also the presence of $h c p$ metallic Co and $f c c \mathrm{CoO}$. After annealing at $700^{\circ} \mathrm{C}$, the $f c c$ Co is the dominant phase with the presence of traces of $\mathrm{CoO}$. The enhancement of the intensity and the narrowing of the fcc peaks in the xrd patterns for the annealed samples is due to an increase of the average particle size and the better crystallinity following the thermal treatment.

The TEM images from the $25 \% \mathrm{CoO} / \mathrm{MWNTs}$ composite material are illustrated in Figure 2. From these images, it is observed that almost all the carbon nanotubes are decorated on the external surface with numerous nanoparticles, and there is no evidence for isolated particles. The majority of the particles have nearly spherical shape with about $10 \mathrm{~nm}$ diameter. Also there are some larger particles with diameter between $20-30 \mathrm{~nm}$. The single crystallinity of the particles was established by the high-resolution TEM (HRTEM) images. The TEM images of the $25 \mathrm{wt} \% \mathrm{CoO} / \mathrm{MWNTs}$ composite material after the thermal treatment at $500^{\circ} \mathrm{C}$ under $5 \% \mathrm{H}_{2} 95 \%$ Ar atmosphere are presented in Figure 3. The average particle size after the thermal treatment is $20 \mathrm{~nm}$.

It is worth to mention that the as-made composite $\mathrm{CoO} / \mathrm{MWNTs}$ materials are well dispersible in nonpolar organic solvents such as hexane. This was probably due to the surface functionalization of probably both carbon nanotubes and $\mathrm{CoO}$ particles by the oleyl amine molecules. Figure 4(a) shows the FT-IR spectrum from the $25 \% \mathrm{CoO} / \mathrm{MWNTs}$ composite material. The spectrum shows strong absorption bands at 2926 and $2850 \mathrm{~cm}^{-1}$ due to the $\mathrm{CH}_{2}$ stretching of the aliphatic chains, whereas a weak peak at $3006 \mathrm{~cm}^{-1}$ is characteristic of the cis $-\mathrm{HC}=\mathrm{CH}$ - arrangement in the oleyl amine. The peak at $1626 \mathrm{~cm}^{-1}$ is due to the $\nu(\mathrm{C}=\mathrm{C})$ stretching mode while the band at $1452 \mathrm{~cm}^{-1}$ is due to the $\mathrm{CH}_{2}$ scissor. The very broad peak in the $3430 \mathrm{~cm}^{-1}$ region is due to the $v(\mathrm{~N}-\mathrm{H})$ stretching mode, and the band around $1550 \mathrm{~cm}^{-1}$ is probably due to the $\mathrm{NH}_{2}$ scissoring mode which because of the shifting at lower wavenumbers $\left(1595 \mathrm{~cm}^{-1}\right.$ in unbonded oleyl amine) suggests that oleylamine is adsorbed with the $\mathrm{NH}_{2}$ group intact. These results strongly indicate that the surface of MWNTs and $\mathrm{CoO}$ nanocrystals are modified with oleyl amine molecules. Figure 4(b) represents the Raman spectrum of the $\mathrm{CoO} / \mathrm{MWNTs}$ material after annealing at $500^{\circ} \mathrm{C}$. It reveals tow bands centered at 1345 ( $\mathrm{D}$ band) and 1595 (G band) $\mathrm{cm}^{-1}$ which are characteristic for multiwall carbon nanotubes and indicate that in the annealed material the carbon nanotubes has still their crystallinity.

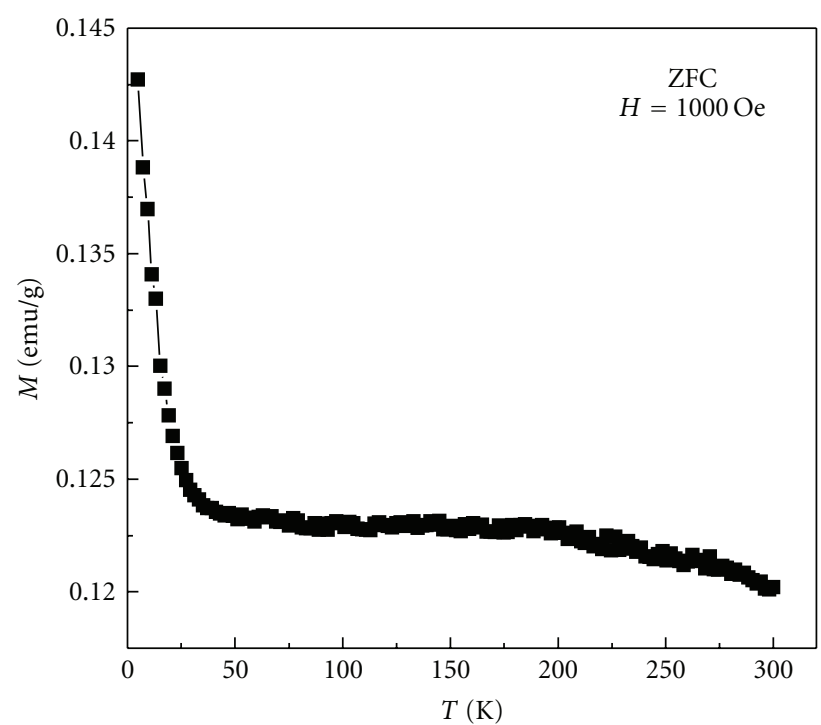

Figure 5: $M$ versus $T$ plot from as-made $25 \mathrm{wt} \% \mathrm{CoO} / \mathrm{MWNTs}$ nanocomposite.

3.2. Magnetic Characterization. The DC magnetic measurements of the samples were carried out using a PPMS (Quantum Design). To perform the zero-field-cooled (ZFC) measurement, the sample was cooled down to $5 \mathrm{~K}$ under zero applied field and then the data were collected applying $1000 \mathrm{Oe}$ field while warming from $5 \mathrm{~K}$ to $300 \mathrm{~K}$. The magnetization $(M)$ versus temperature $(T)$ data in the ZFC mode shows a sharp rise in magnetization at lower temperatures as shown in Figure 5. This trend follows the Curie-Weiss law $(M=C /(T-\theta), C$ and $\theta$ being the Curie constant and Weiss temperature, resp.) for a paramagnetic material. The observed low-temperature Curie tail of the CoO/MWNTs composite (Figure 5) may be attributed to the uncompensated spins $\left(\mathrm{Co}^{2+}\right)$ at the surface of $\mathrm{CoO}$ nanoparticles [20]. It is known that the Weiss temperature $\theta$ is a measure of exchange interaction between spins; a positive (negative) $\theta$ represents ferromagnetic (AFM) interaction [21]. The Weiss temperature was determined by fitting a line to the $1 / M$ versus $T$ data. The straight line was extrapolated to the $T$ axis, and the fitting result shows $\theta=180 \mathrm{~K}$. This is consistent with the expected antiferromagnetic ordering in $\mathrm{CoO}$ NPs.

It is to be noted that bulk $\mathrm{CoO}$ is an antiferromagnet with Néel temperature $\left(T_{\mathrm{N}}\right) \sim 293 \mathrm{~K}$ [22]. However, in this study, the $T_{\mathrm{N}}$ peak is not observable due to the mainly paramagnetic nature of the $\mathrm{CoO}$ nanoparticles [20]. Some recent studies show that the value of $T_{\mathrm{N}}$ may change with variation in size of AF nanoparticles [23]. Néel proposed that antiferromagnetic (AF) nanoparticles may exhibit superparamagnetism and weak ferromagnetism (FM) due to the uncompensated spins on the surface of the nanoparticles [22]. In our sample, the increased magnetization below $50 \mathrm{~K}$ indicates that the surface effect resulting from the increasing number of spins at the surface shell for small size nanoparticles $(<20 \mathrm{~nm})$. This would imply that surface magnetic ordering dominates in this system over the bulk 


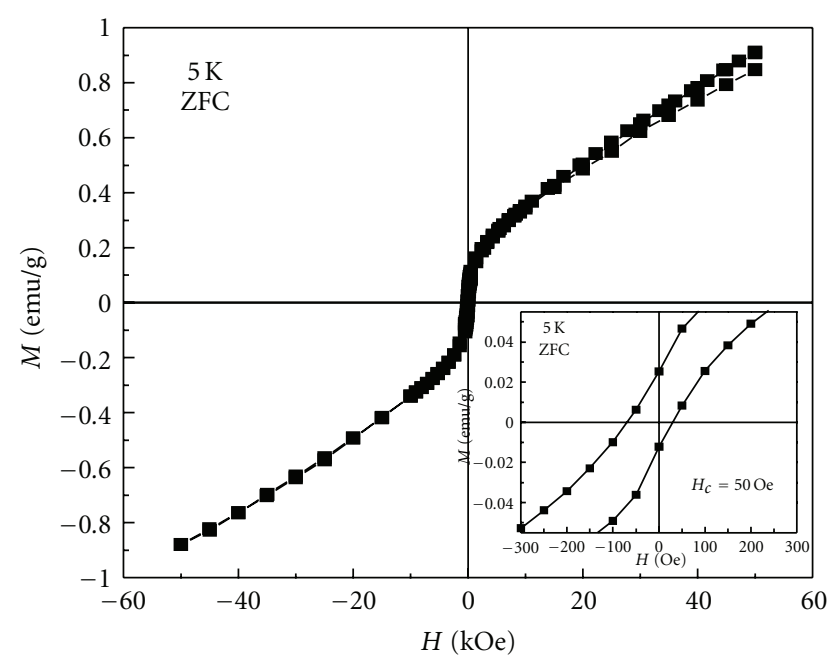

(a)

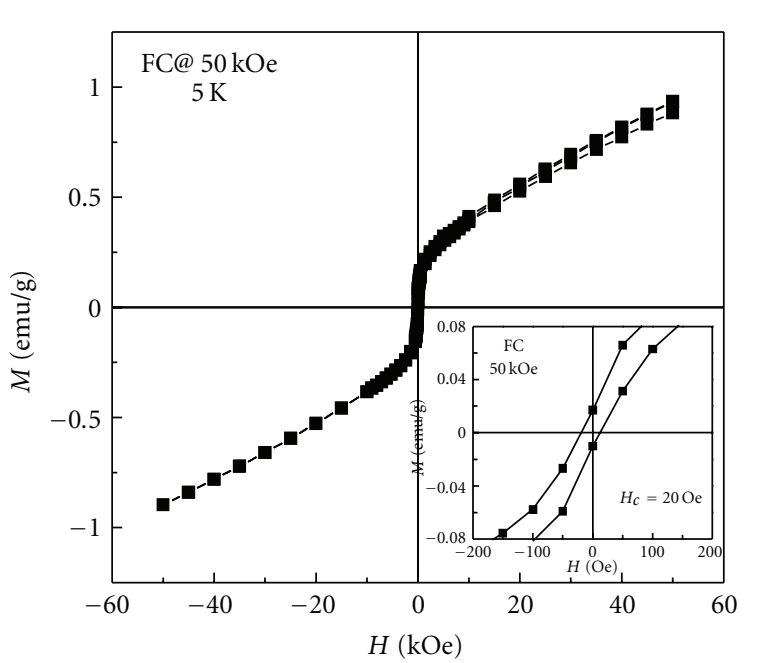

(b)

Figure 6: $M$ versus $H$ plots of $25 \mathrm{wt} \% \mathrm{CoO} / \mathrm{MWNT}$ composite at $5 \mathrm{~K}$ in (a) ZFC and (b) FC mode.

AF ordering in $\mathrm{CoO}$ nanoparticles. Similar observations have been reported for $\mathrm{CoO}$ NPs where the magnetization increases sharply below $100 \mathrm{~K}[24,25]$.

To get a clearer picture about the uncompensated spins and possible interaction between the surface shell and the core of the nanoparticles, we have performed magnetization $(M)$ versus magnetic field $(H)$ measurements. Figures 6(a) and 6(b) show the $M$ versus $H$ data of $\mathrm{CoO} / \mathrm{MWNT}$ under ZFC and FC at high field ( $50 \mathrm{kOe})$ mode at $5 \mathrm{~K}$, respectively. From this measurement, it is clear that magnetization does not saturate even at higher field which is consistent of the paramagnetic or antiferromagnetic nature of $\mathrm{CoO} / \mathrm{MWNTs}$ composite. The positive slope observed at higher fields is caused by the susceptibility of the $\mathrm{CoO}$ shell. In both sets (ZFC-FC) of measurements, the shape of the hysteresis loop can be divided into two parts: an "S"-shaped curve at the lower field region and a linear behavior with positive slope at higher field. The $M-H$ curvature ("S") could be associated with the weak ferromagnetic contribution from the uncompensated surface spin of $\mathrm{CoO}$ nanoparticles. Whereas the linear portion corresponds to the paramagnetic or antiferromagetic $\mathrm{CoO}$ nanoparticles $[24,25]$. This magnetic behavior seems to indicate that the $\mathrm{CoO}$ nanoparticle system can be described as a core-shell structure. A small coercivity observed in this sample indicates that there is a weak ferromagnetic interaction (inset Figures of 6(a) and 6(b)) present at the low temperature. This type of magnetic behavior has been observed in $\mathrm{CoO}$ nanoparticles when the particle size is less than $16 \mathrm{~nm}$ [26]. The above $M-H$ result is also consistent with the results obtained from the $M-T$ measurement.

The room temperature $M$ versus $H$ data were measured for the sample annealed at $500^{\circ} \mathrm{C}$ as shown in Figure 7. The appearance of open hysteresis loop with coercivity $H_{C}=$ 560 Oe clearly indicates the ferromagnetic nature of this sample. The saturation magnetization $M_{s}$ and remanence $M_{r}$ are observed as $8.5 \mathrm{emu} / \mathrm{g}$ and $3.3 \mathrm{emu} / \mathrm{g}$, respectively.

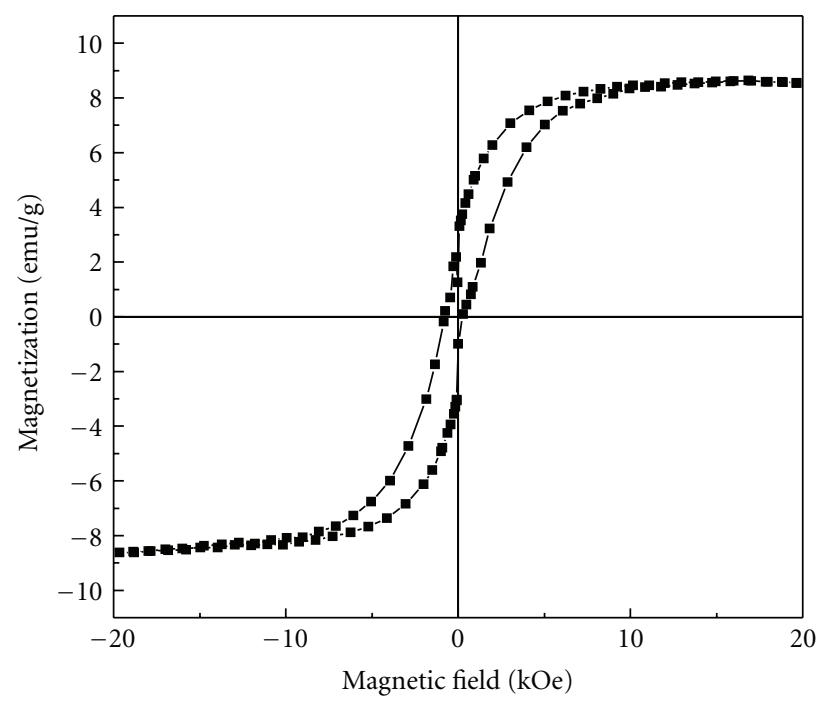

FIGURE 7: Room temperature hysteresis loop from annealed at $500^{\circ} \mathrm{C}, 25 \mathrm{wt} \% \mathrm{CoO} / \mathrm{MWNTs}$ nanocomposite material.

It should be noted that Co/MWNT has higher saturation magnetization compared to $\mathrm{CoO} / \mathrm{MWNTs}$. The higher $M_{s}$ value of $\mathrm{Co} / \mathrm{MWNT}$ reveals that the $\mathrm{CoO} / \mathrm{MWNTs}$ composite has largely transformed into ferromagnetic Co/MWNT composite after high-temperature annealing under reducing atmosphere. Although the magnetization of a nanoparticle is somewhat different from its bulk counterpart, the quite low value of saturation magnetization here indicates that a small quantity of metallic cobalt is present in this composite.

3.3. NMR Characterization. The $\mathrm{CoO}$ or $\mathrm{Co} /$ carbon nanotube materials were further characterized by NMR methods. We have performed NMR measurements on the ${ }^{59} \mathrm{Co}$ 


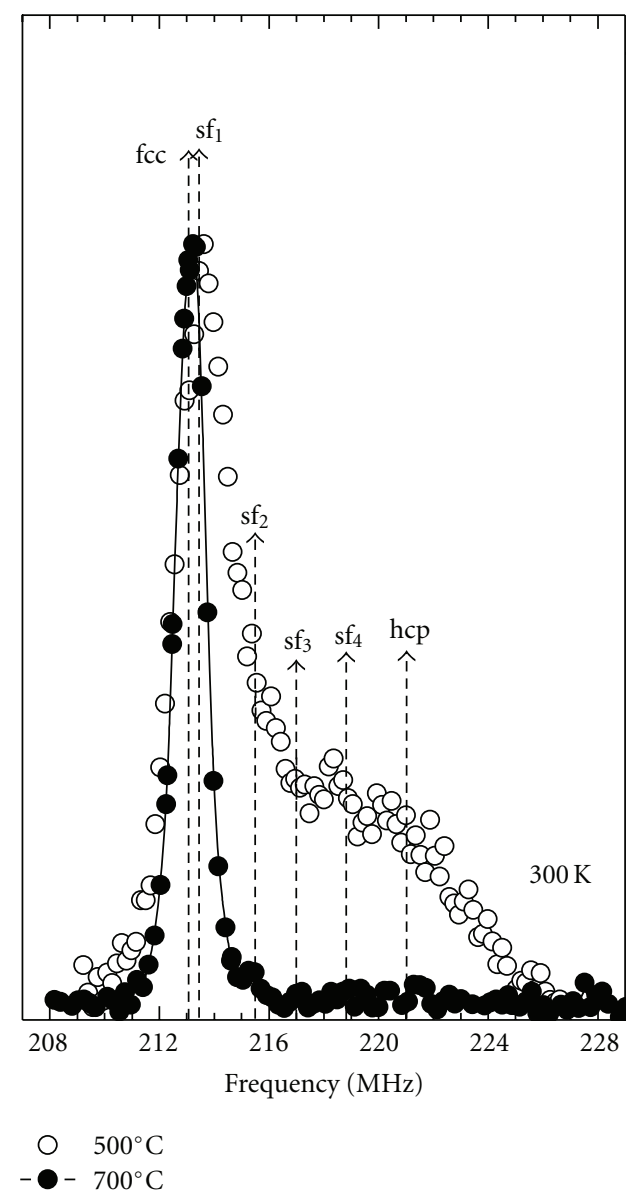

(a)

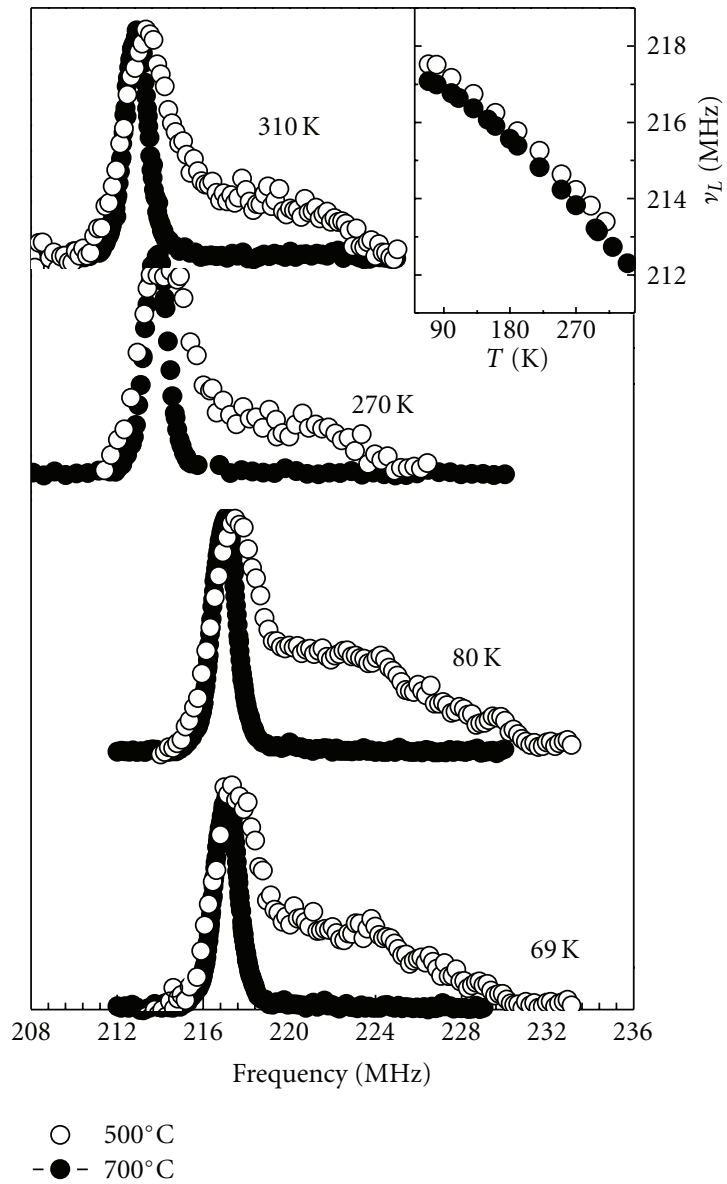

(b)

Figure 8: (a) A comparison between the ${ }^{59} \mathrm{Co}$ NMR spectra for samples annealed at $500{ }^{\circ} \mathrm{C}$ and $700{ }^{\circ} \mathrm{C}$, as measured at $300 \mathrm{~K}$. (b) ${ }^{59} \mathrm{Co} \mathrm{NMR}$ spectra at selected temperatures for both samples. The inset shows the temperature dependence of the Larmor frequency $v_{\mathrm{L}}$, that is, the local magnetic moment, for the unfaulted fcc structure.

nuclei which have natural abundance 100\% (as compared to $1.108 \%$ for ${ }^{13} \mathrm{C}$ nuclei). ${ }^{59} \mathrm{Co}$ NMR spectroscopy gives microscopic information on the structure and the local magnetic properties of the present magnetic materials. Furthermore, here, in contrast to nonmagnetic materials, one can perform zero-field ${ }^{59} \mathrm{Co}$ NMR due to the spontaneous hyperfine fields present at the nuclear sites [27].

We have carried out ${ }^{59} \mathrm{Co}$ spin echo NMR measurements on a home built spectrometer operating in the frequency range $5-600 \mathrm{MHz}$. Due to the large width of the resonance lines, the ${ }^{59} \mathrm{Co}$ (nuclear spin $I=7 / 2$, gyromagnetic ratio $\gamma / 2 \pi=10.0541 \mathrm{MHz} / \mathrm{T})$ NMR spectra were acquired by sweeping the frequency (spin-echo point by point method). At each frequency, a usual $t_{p}-\tau-t_{p}$ pulse sequence was employed and the spin echo amplitude was measured. The typical value of the pulse length was $t_{p}=1 \mu \mathrm{s}$, and the separation between the two rf pulses was $\tau=8 \mu$ s. The nuclear spin-lattice relaxations time $T_{1}$ was acquired by measuring the recovery of the nuclear magnetization following a short sequence of saturating radio frequency pulses. The NMR measurements were carried out in the temperature range 69-340 K by employing an Oxford 1200 continuous flow cryostat.

The zero field ${ }^{59} \mathrm{Co}$ lineshape measurements were performed on the as-made and the annealed samples. In the case of the as-made sample, the observation of the zero field ${ }^{59} \mathrm{Co}$ signal from the $\mathrm{CoO}$ phase, or from a minority metallic phase was not feasible for measurements carried down to $70 \mathrm{~K}$. The as-made sample was then heated under reducing atmosphere at two different treatment temperatures, that is, $500^{\circ} \mathrm{C}$ and $700^{\circ} \mathrm{C}$ for $30 \mathrm{~min}$. The annealing of the samples gives rise to a ferromagnetic component and the observation of the zero field ${ }^{59} \mathrm{Co}$ NMR was feasible for both samples. Figure 8(a) shows the ${ }^{59} \mathrm{Co}$ NMR spectra acquired at $300 \mathrm{~K}$ from the samples annealed at 500 and $700^{\circ} \mathrm{C}$. The comparison of the two samples shows considerable differences. In the case of sample annealed at $500^{\circ} \mathrm{C}$, the ${ }^{59} \mathrm{Co}$ NMR signal spreads from 210 to $225 \mathrm{MHz}$, and a well-resolved peak is observed at $213.3 \mathrm{MHz}$. The position of this peak corresponds to the unfaulted fcc structure of metallic cobalt $(213.1 \mathrm{MHz})$ reported in the literature [28]. The long tail observed at higher frequencies is the fingerprint of different structural 
components coexisting with the fcc structure. Specifically, the unfaulted hcp structure of Co is expected at $221 \mathrm{MHz}$ [29] and is broader than the corresponding fcc peak due to the quadrupolar interactions with the non cubic crystalline field. In the frequency range $213.1-221 \mathrm{MHz}$, the signal is coming from different types of stacking faults $\left(\mathrm{sf}_{1}, \mathrm{sf}_{2}, \mathrm{sf}_{3}\right.$, and $\mathrm{sf}_{4}$ in Figure 8(a)) [30,31]. According to [30,31], the $\mathrm{sf}_{1}(214 \mathrm{MHz})$ and $\mathrm{sf}_{2}(215.5 \mathrm{MHz})$ faults correspond to one stacking error in the $\mathrm{fcc}$ sequence ( $\mathrm{fcc}$ twin faults, or two consecutive hcp deformations). On the other hand, the stacking faults $\mathrm{sf}_{3}(217 \mathrm{MHz})$ and $\mathrm{sf}_{4}(219 \mathrm{MHz})$ correspond to one stacking error in the hcp structure (hcp twin fault, or two stacking faults in fcc). The rf enhancement factor of the NMR signal was found to be the same throughout the ${ }^{59} \mathrm{Co}$ spectra, thus, the spectral areas of the distinct components are representative of their relative ratio. On the other hand, the spectrum of sample annealed at $700^{\circ} \mathrm{C}$ shows a single peak which is considerably narrower (Figure $8(\mathrm{a})$ ). The peak is centered at $213.1 \mathrm{MHz}$ which is the resonance frequency of the unfaulted fcc structure, while no signal is observed at frequencies corresponding to the hcp Co resonance, or to stacking faults. We have continued our experiments at lower temperatures (Figure 8(b)) where the sensitivity is higher and we have found no traces of the hcp structure in sample annealed at $700^{\circ} \mathrm{C}$ for measurements performed to temperatures as low as $69 \mathrm{~K}$. Thus, the thermal treatment at $700^{\circ} \mathrm{C}$ gives rise to phase transformation of the hcp structure to the fcc and also to disappearance of the faulted phases. Similar behavior has also been observed in bulk Co [32] and in polycrystalline cobalt with $\mu \mathrm{m}$ particle size [33]. By decreasing temperature, the NMR peaks move to higher frequency for both samples. The temperature dependence of the Larmor frequency $v_{\mathrm{L}}$ (proportional to the local magnetic moment) for the unfaulted fcc structure is plotted in the insert of Figure $8(\mathrm{~b})$ for both annealed samples. The Tdependence of $v_{\mathrm{L}}$ is the same for both samples and agrees with the reported temperature behavior observed in the bulk Co metal [34].

As we have seen so far, the annealing of the samples under reducing atmosphere leads to important structural modifications which have been probed by NMR lineshape measurements. Significant information was also obtained by ${ }^{59} \mathrm{Co}$ spin-lattice relaxation measurements. The temperature dependence of ${ }^{59} \mathrm{Co}$ spin-lattice relaxation rate, $1 / T_{1}$, is presented in Figure 9. The measurements were performed at the position of the fcc peak. In case of the sample annealed at $500^{\circ} \mathrm{C}$, the spin-lattice relaxation time measurements were also performed along the high-frequency tail of the NMR line but no significant difference in $1 / T_{1}$ was found. For the sample annealed at $700^{\circ} \mathrm{C}$, the relaxation rate $1 / T_{1}$ follows a linear temperature dependence with a slope $(12.8 \pm 0.3) \mathrm{s}^{-1}$. $\mathrm{K}^{-1}$. This linear temperature dependence is commonly found in $3 \mathrm{~d}$ ferromagnetic metals and is attributed to fluctuations of the orbital current of the $d$ band electrons [35]. The value of the slope we have found here is in agreement with the literature $[36,37]$. A different $T$-dependence of $1 / T_{1}$ is observed for the sample annealed at $500^{\circ} \mathrm{C}$, where a broad peak is superimposed on the underlying linear temperature dependence. The peak in $1 / T_{1}$ is representative of magnetic

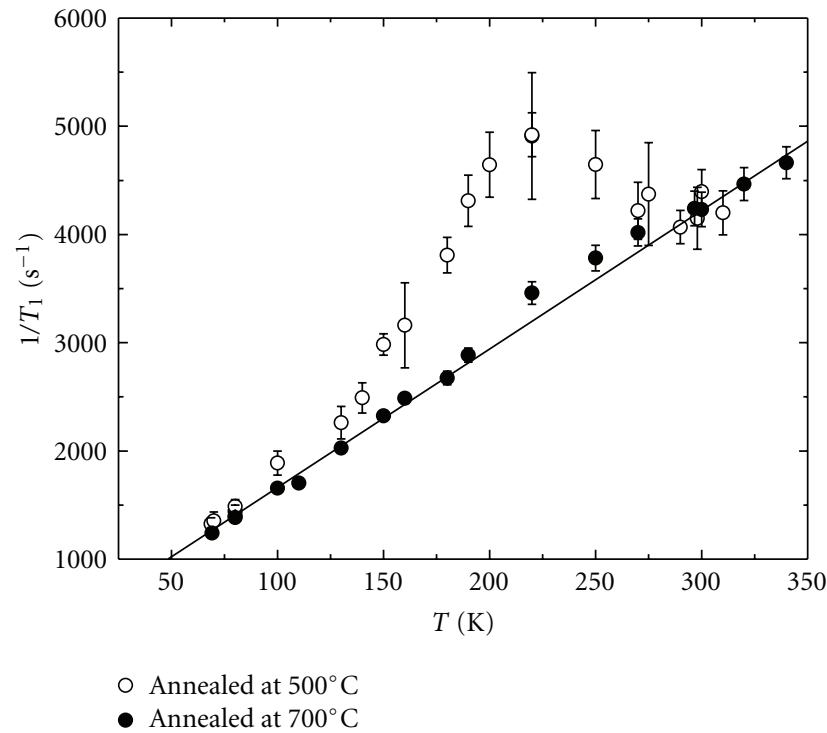

Figure 9: Temperature dependence of the ${ }^{59} \mathrm{Co}$ spin-lattice relaxation rate, $1 / T_{1}$, for nanocomposite samples annealed at $500^{\circ} \mathrm{C}$ and $700^{\circ} \mathrm{C}$.

fluctuations but is uncommon in $3 \mathrm{~d}$ ferromagnetic metals. We note here that the annealing at $500^{\circ} \mathrm{C}$ under reducing atmosphere has lead to partially reduction of $\mathrm{CoO}$ and thus to coexistence of metallic $\mathrm{Co}$ and $\mathrm{CoO}$ phases. Even though the ${ }^{59} \mathrm{Co}$ NMR measurements performed here are for Co nuclei in ferromagnetic metallic regions, the relaxation rate $1 / T_{1}$ can still be sensitive to fluctuations of a coupled system, that is, the uncompensated spins of the $\mathrm{CoO}$ grains. A similar behavior of $1 / T_{1}$ is reported in [38] where the effect of oxidation in the spin-lattice relaxation rate of Co-based ferromagnetic nanowires was studied. The enhancement of the spin-lattice relaxation rate in [38] was attributed to the thermal fluctuations of the uncompensated spins of $\mathrm{CoO}$ grains at the surface of the wires. A similar scenario can be invoked here to explain the enhancement of the relaxation rate in the sample annealed at $500^{\circ} \mathrm{C}$. On the other hand, for the sample annealed at $700^{\circ} \mathrm{C}$, an almost perfect reduction of $\mathrm{CoO}$ has been achieved and the relaxation rate $1 / T_{1}$ is showing the typical linear $T$-dependence.

\section{Conclusions}

Nanocomposite $\mathrm{CoO}$ or $\mathrm{Co} / \mathrm{carbon}$ nanotubes materials were synthesized by one pot chemical route. The structural and chemical phase transformation of CoO/MWNTs composite was investigated using by XRD, TEM, magnetic, and NMR measurements. The magnetic measurements reveal the existence of uncompensated surface spins on $\mathrm{CoO}$ nanoparticles and show weak ferromagnetic interaction at low temperatures. The CoO/MWNTs composite was annealed at $500^{\circ} \mathrm{C}$ under $4 \% \mathrm{H}_{2}-96 \%$ Ar gas mixture resulting partial reduction of CoO. This sample consists of both $\mathrm{CoO}$ and metallic $\mathrm{Co} / \mathrm{MWNT}$ phases. Further annealing at $700^{\circ} \mathrm{C}$ gives complete reduction of $\mathrm{CoO}$ to fcc metallic $\mathrm{Co}$ 
particles. In the sample annealed at $500^{\circ} \mathrm{C}$, the nuclear spinlattice relaxation rate gives evidence for slow fluctuations of the uncompensated surface spins. On the other hand, the nuclear spin-lattice relaxation rate in the sample annealed at $700^{\circ} \mathrm{C}$ follows the typical linear temperature dependence commonly found in ferromagnetic metals. Furthermore, NMR shows that the sample annealed at $500^{\circ} \mathrm{C}$ is a mixture of fcc and hcp phases with a significant percentage of stacking faults. The stacking fault sites cannot be easily probed by $\mathrm{XRD}$. The annealing at $700^{\circ} \mathrm{C}$ gives rise to transformation of the hcp phase to the fcc and simultaneous reduction of the stacking fault content. The magnetic characterization seems to be a complementary technique to probe the structural transformation in a small amount of sample.

\section{Acknowledgments}

M. Belesi acknowledges support by the research project ENTER-2004 that is cofinanced by E.U.-European Social Fund $(75 \%)$ and the Greek Ministry of DevelopmentGSRT (25\%). S. Hariharan and S. Pal thank the Center for Integrated Functional Materials and DoD-USAMRMC for support through Grant no. W81XWH-07-1-0708.

\section{References}

[1] S. Pal, S. Chandra, M.-H. Phan, P. Mukherjee, and H. Srikanth, "Carbon nanostraws: nanotubes filled with superparamagnetic nanoparticles," Nanotechnology, vol. 20, no. 48, Article ID 485604, 2009.

[2] Z. Deng, E. Yenilmez, J. Leu et al., "Metal-coated carbon nanotube tips for magnetic force microscopy," Applied Physics Letters, vol. 85, no. 25, pp. 6263-6265, 2004.

[3] X. Peng, Z. Iuan, Z. Di, Z. Zhang, and C. Zhu, "The addition of mesoporosity to activated carbon fibers by a simple reactivation process," Carbon, vol. 43, no. 4, pp. 855-857, 2005.

[4] S. Utsumi, K. Urita, H. Kanoh et al., "Preparing a magnetically responsive single-wall carbon nanohorn colloid by anchoring magnetite nanoparticles," Journal of Physical Chemistry B, vol. 110, no. 14, pp. 7165-7170, 2006.

[5] V. Georgakilas, D. Gournis, V. Tzitzios, L. Pasquato, D. M. Guldi, and M. Prato, "Decorating carbon nanotubes with metal or semiconductor nanoparticles," Journal of Materials Chemistry, vol. 17, no. 26, pp. 2679-2694, 2007.

[6] J. Wan, W. Cai, J. Feng, X. Meng, and E. Liu, "In situ decoration of carbon nanotubes with nearly monodisperse magnetite nanoparticles in liquid polyols," Journal of Materials Chemistry, vol. 17, no. 12, pp. 1188-1192, 2007.

[7] H. Zhang, N. Du, P. Wu, B. Chen, and D. Yang, "Functionalization of carbon nanotubes with magnetic nanoparticles: general nonaqueous synthesis and magnetic properties," Nanotechnology, vol. 19, no. 13, Article ID 315604, 2008.

[8] A. S. Adekunle and K. I. Ozoemena, "Electron transfer behaviour of single-walled carbon nanotubes electrodecorated with nickel and nickel oxide layers," Electrochimica Acta, vol. 53, no. 19, pp. 5774-5782, 2008.

[9] S. V. Mahajan, S. A. Hasan, J. Cho, M. S. P. Shaffer, A. R. Boccaccini, and J. H. Dickerson, "Carbon nanotube-nanocrystal heterostructures fabricated by electrophoretic deposition," Nanotechnology, vol. 19, no. 19, Article ID 195301, 2008.
[10] H. Orikasa, N. Inokuma, S. Ittisanronnachai, X. H. Wang, O. Kitakami, and T. Kyotani, "Template synthesis of waterdispersible and magnetically responsive carbon nano test tubes," Chemical Communications, no. 19, pp. 2215-2217, 2008.

[11] Q. Liu, W. Ren, Z. G. Chen et al., "Direct synthesis of carbon nanotubes decorated with size-controllable Fe nanoparticles encapsulated by graphitic layers," Carbon, vol. 46, no. 11, pp. 1417-1423, 2008.

[12] S. F. Chin, K. S. Iyer, and C. L. Raston, "Fabrication of carbon nano-tubes decorated with ultra fine superparamagnetic nano-particles under continuous flow conditions," Lab on a Chip, vol. 8, no. 3, pp. 439-442, 2008.

[13] N. Mackiewicz, G. Surendran, H. Remita et al., "Supramolecular self-assembly of amphiphiles on carbon nanotubes: a versatile strategy for the construction of CNT/metal nanohybrids, application to electrocatalysis," Journal of the American Chemical Society, vol. 130, no. 26, pp. 8110-8111, 2008.

[14] D. Wang, Z. C. Li, and L. Chen, "Templated synthesis of singlewalled carbon nanotube and metal nanoparticle assemblies in solution," Journal of the American Chemical Society, vol. 128, no. 47, pp. 15078-15079, 2006.

[15] F. Stoffelbach, A. Aqil, C. Jérome, R. Jérome, and C. Detrembleur, "An easy and economically viable route for the decoration of carbon nanotubes by magnetite nanoparticles, and their orientation in a magnetic field," Chemical Communications, no. 36, pp. 4532-4533, 2005.

[16] C. Gao, W. Li, H. Morimoto, Y. Nagaoka, and T. Maekawa, "Magnetic carbon nanotubes: synthesis by electrostatic selfassembly approach and application in biomanipulations," Journal of Physical Chemistry B, vol. 110, no. 14, pp. 72137220, 2006.

[17] W. Li, C. Gao, H. Qian, J. Ren, and D. Yan, "Multiaminofunctionalized carbon nanotubes and their applications in loading quantum dots and magnetic nanoparticles," Journal of Materials Chemistry, vol. 16, no. 19, pp. 1852-1859, 2006.

[18] C. Hsieh and G. L. Lin, "Enhancement in thermal stability of metal-insulator-metal capacitors for deep trench DRAM application," ECS Transactions, vol. 13, no. 14, pp. 73-89, 2008.

[19] V. Tzitzios, V. Georgakilas, I. Zafiropoulou et al., "A general chemical route for the synthesis of capped nanocrystalline materials," Journal of Nanoscience and Nanotechnology, vol. 8, no. 6, pp. 3117-3122, 2008.

[20] J. B. Tracy, D. N. Weiss, D. P. Dinega, and M. G. Bawendi, "Exchange biasing and magnetic properties of partially and fully oxidized colloidal cobalt nanoparticles," Physical Review B, vol. 72, no. 6, Article ID 064404, 8 pages, 2005.

[21] C. Kittel, Introduction to Solid State Physics, Wiley, New York, NY, USA, 7th edition, 1996.

[22] L. Néel, “Théorie des propriétés magnétiques des grains fins antiferromagnétiques: Superparamagnétism et Superantiferromagnétism," in Low Temperature Physics, C. DeWitt, B. Dreyfus, and P. D. de Gennes, Eds., Gordon and Breach, New York, NY, USA, 1962.

[23] T. Ambrose and C. L. Chien, "Finite-size effects and uncompensated magnetization in thin antiferromagnetic CoO Layers," Physical Review Letters, vol. 76, no. 10, pp. 1743-1746, 1996.

[24] L. Zhang, D. Xue, and C. Gao, "Anomalous magnetic properties of antiferromagnetic $\mathrm{CoO}$ nanoparticles," Journal of Magnetism and Magnetic Materials, vol. 267, no. 1, pp. 111$114,2003$.

[25] H. T. Zhang and X. H. Chen, "Controlled synthesis and anomalous magnetic properties of relatively monodisperse 
CoO nanocrystals," Nanotechnology, vol. 16, no. 10, pp. 22882294, 2005.

[26] M. Ghosh, E. V. Sampathkumaran, and C. N. R. Rao, "Synthesis and magnetic properties of $\mathrm{CoO}$ nanoparticles," Chemistry of Materials, vol. 17, no. 9, pp. 2348-2352, 2005.

[27] R. E. Watson and A. J. Freeman, "Origin of effective fields in magnetic materials," Physical Review, vol. 123, no. 6, pp. 20272047, 1961.

[28] A. C. Gossard and A. M. Portis, "Observation of nuclear resonance in a ferromagnet," Physical Review Letters, vol. 3, no. 4, pp. 164-167, 1959.

[29] Y. Kôi, A. Tsujimura, and T. Kushida, "NMR of Co in ferromagnetic hexagonal cobalt metal," Journal of the Physical Society of Japan, vol. 15, no. 11, p. 2100, 1960.

[30] L. E. Toth and S. F. Ravitz, "Ferromagnetic nuclear resonance in cobalt nuclei in stacking faults and twins," Journal of Physics and Chemistry of Solids, vol. 24, no. 10, pp. 1203-1206, 1963.

[31] J. Sort, S. Surinach, J. S. Munoz et al., "Role of stacking faults in the structural and magnetic properties of ball-milled cobalt," Physical Review B, vol. 68, Article ID 014421, 7 pages, 2003.

[32] C. R. Houska, B. L. Averbach, and M. Cohen, "The cobalt transformation," Acta Metallurgica, vol. 8, no. 2, pp. 81-87, 1960.

[33] R. Speight, A. Wong, P. Ellis et al., " ${ }^{" 59}$ Co NMR study of the allotropic phase transformation in small ferromagnetic cobalt particles," Physical Review B, vol. 79, no. 5, Article ID 054102, 2009.

[34] M. Shaham, J. Barak, U. El-Hanany, and W. W. Warren, "NMR study of the $3 \mathrm{~d}$ ferromagnetic metals: critical region and paramagnetic phase," Physical Review B, vol. 22, no. 11, pp. 5400-5419, 1980.

[35] T. Moriya, "Nuclear magnetic relaxation in ferromagnetic transition metals," Journal of the Physical Society of Japan, vol. 19, pp. 681-687, 1964.

[36] M. Weger, "Longitudinal nuclear magnetic relaxation in ferromagnetic iron, cobalt, and nickel," Physical Review, vol. 128, no. 4, pp. 1505-1511, 1962.

[37] V. Jaccarino, N. Kaplan, R. E. Walstedt, and J. H. Wernick, "Field dependence of nuclear relaxation in ferromagnetic metals," Physics Letters, vol. 23, no. 9, pp. 514-515, 1966.

[38] V. Scarani, H. De Riedmatten, and J. P. Ansermet, "Co nuclear magnetic resonance studies of magnetic excitations in ferromagnetic nanowires," Applied Physics Letters, vol. 76, no. 7, pp. 903-905, 2000. 

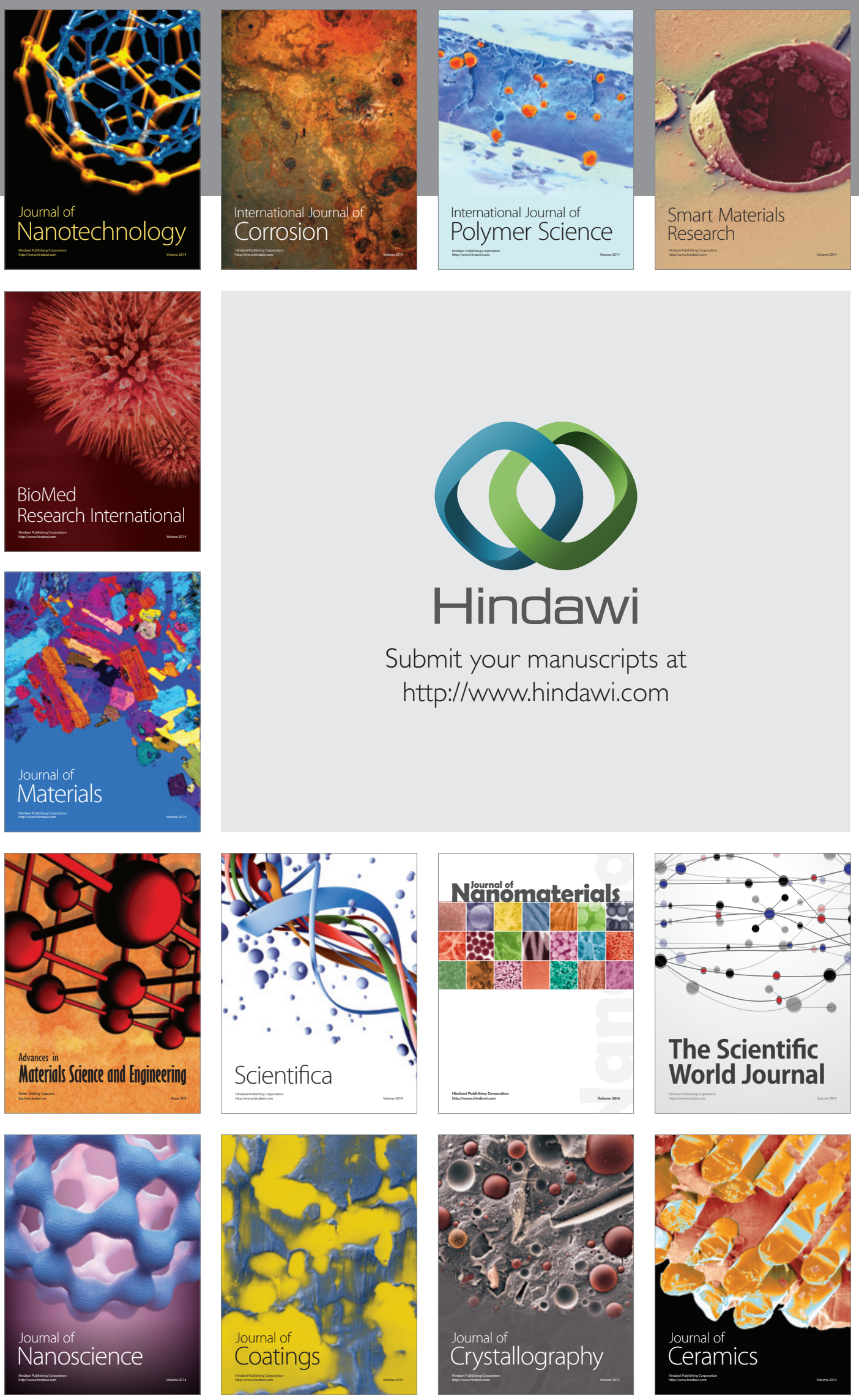

The Scientific World Journal

Submit your manuscripts at

http://www.hindawi.com

\section{World Journal}

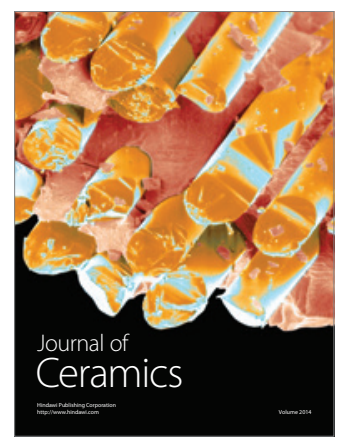

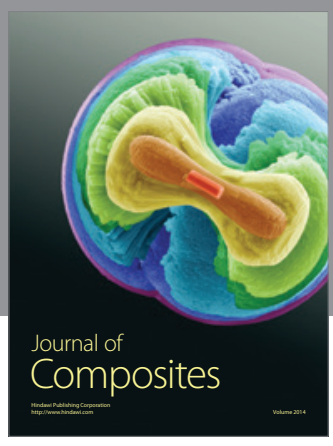
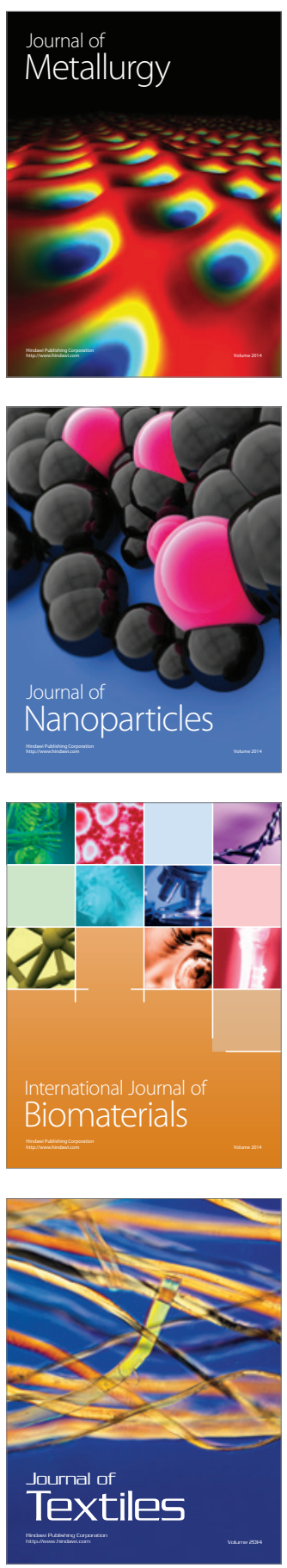